\title{
Greedoids on vertex sets of $B$-joins of graphs
}

\author{
VADIM E. LEVIT and EUGEN MANDRESCU
}

\begin{abstract}
.
Let $\Psi(G)$ be the family of all local maximum stable sets of graph $G$, i.e., $S \in \Psi(G)$ if $S$ is a maximum stable set of the subgraph induced by $S \cup N(S)$, where $N(S)$ is the neighborhood of $S$. It was shown that $\Psi(G)$ is a greedoid for every forest $G$ [15]. The cases of bipartite graphs, triangle-free graphs, and well-covered graphs, were analyzed in [16, 17, $18,19,20,24]$.

If $G_{1}, G_{2}$ are two disjoint graphs, and $B$ is a bipartite graph having $E(B)$ as an edge set and bipartition $\left\{V\left(G_{1}\right), V\left(G_{2}\right)\right\}$, then by $B$-join of $G_{1}, G_{2}$ we mean the graph $B\left(G_{1}, G_{2}\right)$ whose vertex set is $V\left(G_{1}\right) \cup V\left(G_{2}\right)$ and edge set is $E\left(G_{1}\right) \cup E\left(G_{2}\right) \cup E(B)$.

In this paper we present several necessary and sufficient conditions for $\Psi\left(B\left(G_{1}, G_{2}\right)\right)$ to form a greedoid, an antimatroid, and a matroid, in terms of $\Psi\left(G_{1}\right), \Psi\left(G_{2}\right)$ and $E(B)$.
\end{abstract}

\section{REFERENCES}

[1] Björner, A. and Ziegler, G. M., Introduction to greedoids, in N. White (ed.), Matroid Applications, 284-357, Cambridge University Press, 1992

[2] Boros, E., Golumbic, M. C. and Levit, V. E., On the number of vertices belonging to all maximum stable sets of a graph, Discrete Applied Mathematics, 124 (2002), 17-25

[3] Cheston, G. A, Hare, E. O., Hedetniemi, S. T. and Laskar, R. C., Simplicial graphs, Congressus Numerantium, 67 (1988), 105-113

[4] DeAlba, L. M., Some results on minimum skew zero forcing sets, and skew zero forcing number, arXiv:1404.1618 [math.CO] (2014) 13 pp

[5] Deming, R. W., Independence numbers of graphs - an extension of the König-Egerváry theorem, Discrete Mathematics, 27 (1979), 23-33

[6] Edholm, C. J., Hogben, L., Huynh, M., LaGrange, J. and Rowb, D. D., Vertex and edge spread of zero forcing number, maximum nullity, and minimum rank of a graph, Linear Algebra and its Applications, 436 (2012), 4352-4372

[7] Egerváry, E., On combinatorial properties of matrices, Matematikai Lapok, 38 (1931), 16-28

[8] Foldes, S. and Hammer, P. L., Split graphs, Congressus Numerantium, 19 (1977) 311-315

[9] Golumbic, M. C., Hirst, T. and Lewenstein, M., Uniquely restricted matchings, Algorithmica, 31 (2001), 139-154

[10] Hopkins, G. and Staton, W., Graphs with unique maximum independent sets, Discrete Mathematics, 57 (1985), 245-251

[11] Korte, B., Lovász, L. and Schrader, R., Greedoids, Springer-Verlag, Berlin, 1991

[12] König, D., Graphen und Matrizen, Matematikai Lapok, 38 (1931), 116-119

[13] Levit, V. E. and Mandrescu, E., Unicycle bipartite graphs with only uniquely restricted maximum matchings, in Proceedings of the Third International Conference on Combinatorics, Computability and Logic, (DMTCS'1), Springer, (C. S. Calude, M. J. Dinneen and S. Sburlan eds.) (2001), 151-158

[14] Levit, V. E. and Mandrescu, E., Combinatorial properties of the family of maximum stable sets of a graph, Discrete Applied Mathematics, 117 (2002), 149-161

[15] Levit, V. E. and Mandrescu, E., A new greedoid: the family of local maximum stable sets of a forest, Discrete Applied Mathematics, 124 (2002), 91-101

Received: 31.10.2013; In revised form: 09.09.2014; Accepted: 20.10.2014

2010 Mathematics Subject Classification. 05C69, 05C70, 05 B35.

Key words and phrases. Local maximum stable set, maximum matching, uniquely restricted matching, greedoid, antimatroid, matroid.

Corresponding author: Vadim E. Levit; levitv@ariel.ac.il 
[16] Levit, V. E. and Mandrescu, E., Local maximum stable sets in bipartite graphs with uniquely restricted maximum matchings, Discrete Applied Mathematics, 132 (2004), 163-174

[17] Levit, V. E. and Mandrescu, E., Triangle-free graphs with uniquely restricted maximum matchings and their corresponding greedoids, Discrete Applied Mathematics, 155 (2007), 2414-2425

[18] Levit, V. E. and Mandrescu, E., On local maximum stable sets of the corona of a path with complete graphs, in Proceedings of $6^{\text {th }}$ Congress of Romanian Mathematicians, University of Bucharest, Romania, (2009), 565-569

[19] Levit, V. E. and Mandrescu, E., Well-covered graphs and greedoids, in: J. Harland and P. Manyem (Eds.), Theory of Computing 2008. Proceedings of the Fourteenth Computing: The Australasian Theory Symposium (CATS2008), Wollongong, Australia, Conferences in Research and Practice in Information Technology, Vol. 77 (2008) 89-94

[20] Levit, V. E. and Mandrescu, E., The clique corona operation and greedoids, Combinatorial Optimization and Applications, Second International Conference, COCOA 2008, LNCS 5165 (2008), 384-392

[21] Levit, V. E. and Mandrescu, E., Greedoids on the vertex set of a unicycle graph, Congressus Numerantium, 197 (2009), 183-191

[22] Levit, V. E. and Mandrescu, E., Graph operations that are good for greedoids, Discrete Applied Mathematics, 158 (2010), 1418-1423

[23] Levit, V. E. and Mandrescu, E., On local maximum stable set greedoids, Discrete Mathematics, 312 (2012), 588-96

[24] Levit, V. E. and Mandrescu, E., Local maximum stable set greedoids stemming from very well-covered graphs, Discrete Applied Mathematics, 160 (2012), 1864-1871

[25] Monshizadeh, N., Zhangy, S. and Camlibel, M. K., Zero forcing sets and controllability of dynamical systems defined on graphs, IEEE Transactions on Automatic Control (2014), (in press) doi: 10.1109/TAC.2014.2308619

[26] Nemhauser, G. L. and Trotter, Jr., L. E., Vertex packings: structural properties and algorithms, Mathematical Programming, 8 (1975), 232-248

[27] Sterboul, F., A characterization of the graphs in which the transversal number equals the matching number, Journal of Combinatorial Theory Series B, 27 (1979), 228-229

[28] Trefois, M. and Delvenne, J. C., Zero forcing number, constraint matchings and strong structural controllability, arXiv:1405.6222 [cs.DM] (2014) 19 pp

ARIEL UNIVERSITY

Department OF COMPUTER SCIENCE AND MATHEMATICS

KIRYAT HAMADA, 40700, ARIEL, ISRAEL

E-mail address: levitveariel.ac.il

Holon Institute of TeChNOlogy

DEPARTMENT OF COMPUTER SCIENCE

52 GolOMB ST., 58102, HolON, IsRAEL

E-mail address: eugen_mehit.ac.il 